\title{
Density of the set of probability measures with the martingale representation property
}

\author{
Dmitry Kramkov* Sergio Pulido ${ }^{\dagger}$
}

July 8, 2019

\begin{abstract}
Let $\psi$ be a multi-dimensional random variable. We show that the set of probability measures $\mathbb{Q}$ such that the $\mathbb{Q}$-martingale $S_{t}^{\mathbb{Q}}=$ $\mathbb{E}^{\mathbb{Q}}\left[\psi \mid \mathcal{F}_{t}\right]$ has the Martingale Representation Property (MRP) is either empty or dense in $\mathcal{L}_{\infty}$-norm. The proof is based on a related result involving analytic fields of terminal conditions $(\psi(x))_{x \in U}$ and probability measures $(\mathbb{Q}(x))_{x \in U}$ over an open set $U$. Namely, we show that the set of points $x \in U$ such that $S_{t}(x)=\mathbb{E}^{\mathbb{Q}(x)}\left[\psi(x) \mid \mathcal{F}_{t}\right]$ does not have the MRP, either coincides with $U$ or has Lebesgue measure zero. Our study is motivated by the problem of endogenous completeness in financial economics.
\end{abstract}

Keywords: martingale representation property, martingales, stochastic integrals, analytic fields, endogenous completeness, complete market, equilibrium.

AMS Subject Classification (2010): 60G44, 60H05, 91B51, $91 \mathrm{G} 99$.

\footnotetext{
${ }^{*}$ Carnegie Mellon University, Department of Mathematical Sciences, 5000 Forbes Avenue, Pittsburgh, PA, 15213-3890, US. The author also holds a part-time position at the University of Oxford. Email: kramkov@cmu.edu

†Laboratoire de Mathématiques et Modélisation d'Évry (LaMME), Université d'ÉvryVal-d'Essonne, ENSIIE, Université Paris-Saclay, UMR CNRS 8071, IBGBI 23 Boulevard de France, 91037 Évry Cedex, France. Email: sergio.pulidonino@ensiie.fr. The author's research benefited from the support of the Chair Markets in Transition (Fédération Bancaire Française) and the project ANR 11-LABX-0019.
} 


\section{Introduction}

Let $\left(\Omega, \mathcal{F},\left(\mathcal{F}_{t}\right), \mathbb{P}\right)$ be a filtered probability space, $\mathbb{Q}$ be an equivalent probability measure, and $S=\left(S_{t}^{i}\right)$ be a multi-dimensional martingale under $\mathbb{Q}$. It is often important to know whether $S$ has the Martingale Representation Property (MRP), that is, whether every local martingale under $\mathbb{Q}$ is a stochastic integral with respect to $S$. For instance, in mathematical finance such MRP corresponds to the completeness of the market with stock prices $S$. By Jacod's theorem, $S$ has the MRP if and only if $\mathbb{Q}$ is its only equivalent martingale measure.

In many applications, $S$ is defined in a forward form, as a solution of an $\mathrm{SDE}$, and the verification of the MRP is quite straightforward. Suppose, for example, that $S$ is a $d$-dimensional Itô process such that

$$
d S_{t}=\sigma_{t}\left(\alpha_{t} d t+d B_{t}\right)
$$

where $B$ is a $d$-dimensional Brownian motion, $\alpha=\left(\alpha_{t}\right)$ is a $d$-dimensional market price of risk process and $\sigma=\left(\sigma_{t}\right)$ is a $d \times d$-dimensional volatility process. Let us assume that the local martingale

$$
Z_{t}=\exp \left(-\int_{0}^{t} \alpha_{s} d B_{s}-\frac{1}{2} \int_{0}^{t}\left|\alpha_{s}\right|^{2} d s\right), \quad t \geq 0
$$

is uniformly integrable; this fact can usually be verified by Novikov's or Kazamaki's conditions. By Girsanov's theorem, $Z$ is the density process of an equivalent martingale measure $\mathbb{Q}$ for $S$. If the filtration is generated by $B$, then $S$ has the MRP (equivalently, $\mathbb{Q}$ is its only equivalent martingale measure) if and only if the matrix-valued volatility process $\sigma=\left(\sigma_{t}\right)$ has full rank $d \mathbb{P} \times d t$ almost surely.

We are interested in the situation where both $S$ and $Z$ are described in a backward form through their terminal values:

$$
\begin{aligned}
Z_{\infty} & =\frac{d \mathbb{Q}}{d \mathbb{P}}=\frac{\zeta}{\mathbb{E}[\zeta]}, \\
S_{t} & =\mathbb{E}^{\mathbb{Q}}\left[\psi \mid \mathcal{F}_{t}\right], \quad t \geq 0,
\end{aligned}
$$

where $\zeta>0$ and $\psi=\left(\psi^{i}\right)$ are given random variables. Such setup naturally arises in the problem of endogenous completeness of financial economics, where the random variable $\psi$ represents the terminal values of the traded 
securities and $\mathbb{Q}$ defines an equilibrium pricing measure. The term "endogenous" indicates that the stock prices $S=\left(S^{i}\right)$ are computed by (1) as part of the solution. The examples include the construction of Radner equilibrium $[1,4,10,6]$ and the verification of the completeness property for a market with options $[2,11]$.

The main focus of the existing literature has been on the case when the random variables $\zeta$ and $\psi$ are defined in terms of a Markov diffusion in a form consistent with Feynman-Kac formula. The proofs have relied on PDE methods and, in particular, on the theory of analytic semigroups [7]. A key role has been played by the assumption that time-dependencies are analytic.

In this paper we do not impose any conditions on the form of the random variables $\zeta$ and $\psi$. Our main results are stated as Theorems 2.3 and 3.1. In Theorem 2.3 we show that the set

$$
\mathcal{Q}(\psi) \triangleq\left\{\mathbb{Q} \sim \mathbb{P}: S_{t}^{\mathbb{Q}} \triangleq \mathbb{E}^{\mathbb{Q}}\left[\psi \mid \mathcal{F}_{t}\right] \text { has the } \mathrm{MRP}\right\}
$$

is either empty or $\mathcal{L}_{\infty}$-dense in the set of all equivalent probability measures. In Theorem 3.1 we consider analytic fields of probability measures $(\mathbb{Q}(x))_{x \in U}$ and terminal conditions $(\psi(x))_{x \in U}$ over an open set $U$. We prove that the exception set

$$
I \triangleq\left\{x \in U: S_{t}(x) \triangleq \mathbb{E}^{\mathbb{Q}(x)}\left[\psi(x) \mid \mathcal{F}_{t}\right] \text { does not have the MRP }\right\}
$$

either coincides with $U$ or has Lebesgue measure zero.

We expect the results of this paper to be useful in problems of financial economics involving the endogenous completeness property. One such application, to the problem of optimal investment under price impact, is discussed in Remark 2.5.

\section{Density of the set of probability measures with the MRP}

We work on a filtered probability space $\left(\Omega, \mathcal{F},\left(\mathcal{F}_{t}\right)_{t \geq 0}, \mathbb{P}\right)$ satisfying the usual conditions of completeness and right-continuity; the initial $\sigma$-algebra $\mathcal{F}_{0}$ is trivial and $\mathcal{F}=\mathcal{F}_{\infty}$. We denote by $\mathcal{L}_{1}=\mathcal{L}_{1}\left(\mathbf{R}^{d}\right)$ and $\mathcal{L}_{\infty}=\mathcal{L}_{\infty}\left(\mathbf{R}^{d}\right)$ the Banach spaces of (equivalence classes of) $d$-dimensional random variables $\xi$ with the norms $\|\xi\|_{\mathcal{L}_{1}} \triangleq \mathbb{E}[|\xi|]$ and $\|\xi\|_{\mathcal{L}_{\infty}} \triangleq \inf \{c>0: \mathbb{P}[|\xi| \leq c]=1\}$. 
We use the same notation $\mathcal{L}_{1}$ for the isometric Banach space of uniformly integrable martingales $M$ with the norm $\|M\|_{\mathcal{L}_{1}} \triangleq\left\|M_{\infty}\right\|_{\mathcal{L}_{1}}$.

For a matrix $A=\left(A^{i j}\right)$ we denote its transpose by $A^{*}$ and define its norm as

$$
|A| \triangleq \sqrt{\operatorname{tr} A A^{*}}=\sqrt{\sum_{i, j}\left|A^{i j}\right|^{2}} .
$$

If $X$ is a $m$-dimensional semimartingale and $\gamma$ is a $m \times n$-dimensional $X$ integrable predictable process, then $\gamma \cdot X=\int \gamma^{*} d X$ denotes the $n$-dimensional stochastic integral of $\gamma$ with respect to $X$. We recall that a $n \times k$-dimensional predictable process $\zeta$ is $(\gamma \cdot X)$-integrable if and only if $\gamma \zeta$ is $X$-integrable. In this case, $\zeta \cdot(\gamma \cdot X)=(\gamma \zeta) \cdot X$ is a $k$-dimensional semimartingale.

Definition 2.1. Let $\mathbb{Q}$ be an equivalent probability measure $(\mathbb{Q} \sim \mathbb{P})$ and $S$ be a $d$-dimensional local martingale under $\mathbb{Q}$. We say that $S$ has the Martingale Representation Property (MRP) if every local martingale $M$ under $\mathbb{Q}$ is a stochastic integral with respect to $S$, that is, there is a predictable $S$-integrable process $\gamma$ with values in $\mathbf{R}^{d}$ such that

$$
M=M_{0}+\gamma \cdot S \text {. }
$$

Remark 2.2. Jacod's theorem in [5, Section XI.1(a)] states that $S$ has the MRP if and only if there is only one $\mathbb{Q} \sim \mathbb{P}$ such that $S$ is a local martingale under $\mathbb{Q}$. Thus, there is no need to mention $\mathbb{Q}$ in the definition of the MRP.

Let $\psi=\left(\psi^{i}\right)_{i=1, \ldots, d}$ be a $d$-dimensional random variable. We denote by $\mathcal{Q}(\psi)$ the family of probability measures $\mathbb{Q} \sim \mathbb{P}$ such that $\mathbb{E}^{\mathbb{Q}}[|\psi|]<\infty$ and the $\mathbb{Q}$-martingale

$$
S_{t}^{\mathbb{Q}}=\mathbb{E}^{\mathbb{Q}}\left[\psi \mid \mathcal{F}_{t}\right], \quad t \geq 0,
$$

has the MRP.

This is our first main result.

Theorem 2.3. Suppose that $\psi \in \mathcal{L}_{1}\left(\mathbf{R}^{d}\right)$ and $\mathcal{Q}(\psi) \neq \emptyset$. Then for every $\epsilon>0$ there is $\mathbb{Q} \in \mathcal{Q}(\psi)$ such that

$$
\left\|\frac{d \mathbb{Q}}{d \mathbb{P}}-1\right\|_{\mathcal{L}_{\infty}} \leq \epsilon .
$$

The proof is based on Theorem 3.1 from Section 3 and on the following elementary lemma. We recall the definition of an analytic function with values in a Banach space at the beginning of Section 3. 
Lemma 2.4. Let $\zeta$ be a nonnegative random variable. Then the map $x \mapsto$ $e^{-x \zeta}$ from $(0, \infty)$ to $\mathcal{L}_{\infty}$ is analytic.

Proof. Fix $y>0$. For every $\omega \in \Omega$ the function $x \mapsto e^{-x \zeta(\omega)}$ has a Taylor's expansion

$$
e^{-x \zeta(\omega)}=\sum_{n=0}^{\infty} A_{n}(y)(\omega)(x-y)^{n}, \quad x \in \mathbf{R},
$$

where

$$
A_{n}(y)=\left.\frac{1}{n !} \frac{d^{n}}{d x^{n}}\left(e^{-x \zeta}\right)\right|_{x=y}=\frac{1}{n !}(-1)^{n} \zeta^{n} e^{-y \zeta} .
$$

We deduce that

$$
\left\|A_{n}(y)\right\|_{\mathcal{L}_{\infty}} \leq \frac{1}{n !} \max _{t \geq 0}\left(t^{n} e^{-y t}\right)=\frac{1}{n !}\left(\frac{n}{e y}\right)^{n} \leq K \frac{1}{\sqrt{n}}\left(\frac{1}{y}\right)^{n},
$$

where the existence of a constant $K>0$ follows from Stirling's formula:

$$
\lim _{n \rightarrow \infty} \frac{\sqrt{2 \pi n}}{n !}\left(\frac{n}{e}\right)^{n}=1 .
$$

It follows that the series in (2) converges in $\mathcal{L}_{\infty}$ provided that $|x-y|<y$.

Proof of Theorem 2.3. We take $\mathbb{R} \in \mathcal{Q}(\psi)$, denote $\zeta \triangleq \frac{d \mathbb{R}}{d \mathbb{P}}$, and for $x>0$ define the random variables

$$
\begin{aligned}
& \zeta(x) \triangleq \frac{1-e^{-x \zeta}}{x}+\frac{x}{1+x}, \\
& \xi(x) \triangleq \zeta(x) \psi,
\end{aligned}
$$

and a probability measure $\mathbb{Q}(x)$ such that

$$
\frac{d \mathbb{Q}(x)}{d \mathbb{P}}=\frac{\zeta(x)}{\mathbb{E}[\zeta(x)]} .
$$

We set $\zeta(0) \triangleq \zeta, \xi(0) \triangleq \zeta \psi$, and $\mathbb{Q}(0) \triangleq \mathbb{R}$ and observe that for every $\omega \in \Omega$ the functions $x \mapsto \zeta(x)(\omega)$ and $x \mapsto \xi(x)(\omega)$ on $[0, \infty)$ are continuous. Since

$$
|\zeta(x)| \leq \zeta \sup _{t \geq 0} \frac{1-e^{-t}}{t}+\frac{x}{1+x} \leq \zeta+1,
$$

the dominated convergence theorem yields that $x \mapsto \zeta(x)$ and $x \mapsto \xi(x)$ are continuous maps from $[0, \infty)$ to $\mathcal{L}_{1}$. By Lemma $2.4, x \mapsto \zeta(x)$ is an analytic 
map from $(0, \infty)$ to $\mathcal{L}_{\infty}$ and thus $x \mapsto \zeta(x)$ and $x \mapsto \xi(x)$ are analytic maps from $(0, \infty)$ to $\mathcal{L}_{1}$. Theorem 3.1 then implies that the exception set

$$
I \triangleq\{x>0: \mathbb{Q}(x) \notin \mathcal{Q}(\psi)\}
$$

is at most countable.

Choose now any $\epsilon>0$. Since

$$
-\frac{1}{1+x} \leq \zeta(x)-1 \leq \frac{1}{x}-\frac{1}{1+x}
$$

there is $x_{0}=x_{0}(\epsilon)$ such that the assertion of the theorem holds for every $\mathbb{Q}(x)$ with $x \geq x_{0}$ and $x \notin I$.

Remark 2.5. Theorem 2.3 plays a key role in our work, in progress, on the problem of optimal investment in a "backward" model of price impact $[3,8]$. There are a large investor with utility function $U=U(x)$ and initial capital $X_{0}$ and a market maker with exponential utility function

$$
V(y)=\frac{1}{a}\left(1-e^{-a y}\right), \quad y \in \mathbf{R}
$$

where $a>0$ is the absolute risk-aversion coefficient. The investor looks for a predictable process $\gamma=\left(\gamma_{t}\right)$ of the numbers of stocks that maximizes the expected utility:

$$
u\left(X_{0}\right)=\sup _{\gamma} \mathbb{E}\left[U\left(X_{0}+\gamma \cdot S(\gamma)_{T}\right)\right]
$$

While the terminal stock prices are fixed to random dividends $\psi$ :

$$
S_{T}(\gamma)=\psi
$$

their intermediate values are set so that the opposite position to the demand $\gamma$ is optimal for the market maker:

$$
-\gamma=\underset{\zeta}{\arg \max } \mathbb{E}\left[V\left(\zeta \cdot S(\gamma)_{T}\right)\right]
$$

The standard first-order conditions in optimal investment lead to the expression for prices $S(\gamma)$ in a backward form:

$$
S_{t}(\gamma)=\mathbb{E}^{\mathbb{Q}(\gamma)}\left[\psi \mid \mathcal{F}_{t}\right]
$$


where

$$
\frac{d \mathbb{Q}(\gamma)}{d \mathbb{P}}=\frac{V^{\prime}\left(-\gamma \cdot S(\gamma)_{T}\right)}{\mathbb{E}\left[V^{\prime}\left(-\gamma \cdot S(\gamma)_{T}\right)\right]}=\frac{\exp \left(a \gamma \cdot S(\gamma)_{T}\right)}{\mathbb{E}\left[\exp \left(a \gamma \cdot S(\gamma)_{T}\right)\right]}
$$

Theorem 2.3 allows us to relax this apparently complex stochastic control problem into a simple static framework. More precisely, we show that

$$
u\left(X_{0}\right)=\max _{\xi \in \mathcal{C}} \mathbb{E}\left[U\left(X_{0}+\xi\right)\right]
$$

where $\mathcal{C}$ is the family of random variables given by

$$
\mathcal{C} \triangleq\left\{\xi: \mathbb{E}\left[\xi V^{\prime}(-\xi)\right]=0\right\}=\left\{\xi: \mathbb{E}\left[\xi e^{a \xi}\right]=0\right\}
$$

The main ingredient of the proof is the assertion that the family of terminal gains of trading strategies

$$
\mathcal{D} \triangleq\left\{\xi: \xi=\gamma \cdot S(\gamma)_{T} \text { for some demand } \gamma\right\}
$$

is $\mathcal{L}_{\infty}$-dense in $\mathcal{C}$, which can be interpreted as an approximate completeness of the model. This claim follows from Theorem 2.3, after we observe that a random variable $\xi \in \mathcal{C}$ also belongs to $\mathcal{D}$ if the $\mathbb{Q}(\xi)$-martingale $S(\xi)$ has the MRP, where

$$
\begin{aligned}
S_{t}(\xi) & =\mathbb{E}^{\mathbb{Q}(\xi)}\left[\psi \mid \mathcal{F}_{t}\right] \\
\frac{d \mathbb{Q}(\xi)}{d \mathbb{P}} & =\frac{V^{\prime}(-\xi)}{\mathbb{E}\left[V^{\prime}(-\xi)\right]}=\frac{\exp (a \xi)}{\mathbb{E}[\exp (a \xi)]}
\end{aligned}
$$

\section{The MRP for analytic fields of martingales}

Let $\mathbf{X}$ be a Banach space and $U$ be an open connected set in $\mathbf{R}^{d}$. We recall that a map $x \mapsto X(x)$ from $U$ to $\mathbf{X}$ is analytic if for every $y \in U$ there exist a number $\epsilon=\epsilon(y)>0$ and elements $\left(Y_{\alpha}(y)\right)$ in $\mathbf{X}$ such that the $\epsilon$-neighborhood of $y$ belongs to $U$ and

$$
X(x)=\sum_{\alpha} Y_{\alpha}(y)(x-y)^{\alpha}, \quad|y-x|<\epsilon .
$$

Here the series converges in the norm $\|\cdot\|_{\mathbf{X}}$ of $\mathbf{X}$, the summation is taken with respect to multi-indices $\alpha=\left(\alpha_{1}, \ldots, \alpha_{d}\right) \in \mathbf{Z}_{+}^{l}$ of non-negative integers, and if $x=\left(x_{1}, \ldots, x_{d}\right) \in \mathbf{R}^{d}$, then $x^{\alpha} \triangleq \prod_{i=1}^{d} x_{i}^{\alpha_{i}}$.

This is our second main result. 
Theorem 3.1. Let $U$ be an open connected set in $\mathbf{R}^{l}$ and suppose that the point $x_{0} \in \mathbf{R}^{l}$ belongs to the closure of $U$. Let $x \mapsto \zeta(x)$ and $x \mapsto \xi(x)$ be continuous maps from $U \cup\left\{x_{0}\right\}$ to $\mathcal{L}_{1}(\mathbf{R})$ and $\mathcal{L}_{1}\left(\mathbf{R}^{d}\right)$, respectively, whose restrictions to $U$ are analytic. For every $x \in U \cup\left\{x_{0}\right\}$, assume that $\zeta(x)>0$ and define a probability measure $\mathbb{Q}(x)$ and a $\mathbb{Q}(x)$-martingale $S(x)$ by

$$
\frac{d \mathbb{Q}(x)}{d \mathbb{P}}=\frac{\zeta(x)}{\mathbb{E}[\zeta(x)]}, \quad S_{t}(x)=\mathbb{E}^{\mathbb{Q}(x)}\left[\frac{\xi(x)}{\zeta(x)} \mid \mathcal{F}_{t}\right] .
$$

If the $\mathbb{Q}\left(x_{0}\right)$-martingale $S\left(x_{0}\right)$ has the $M R P$, then the exception set

$$
I \triangleq\{x \in U: \text { the } \mathbb{Q}(x) \text {-martingale } S(x) \text { does not have the } M R P\}
$$

has Lebesgue measure zero. If, in addition, $U$ is an interval in $\mathbf{R}$, then the set $I$ is at most countable.

The following example shows that any countable set $I$ in $\mathbf{R}$ can play the role of the exception set of Theorem 3.1. In this example we choose $\zeta(x)=1$ (so that $\mathbb{Q}(x)=\mathbb{P}$ ) and take $x \mapsto \xi(x)$ to be a linear map from $\mathbf{R}$ to $\mathcal{L}_{\infty}(\mathbf{R})$.

Example 3.2. Let $\left(\Omega, \mathcal{F},\left(\mathcal{F}_{n}\right), \mathbb{P}\right)$ be a filtered probability space, where the filtration is generated by independent Bernoulli random variables $\left(\epsilon_{n}\right)$ with

$$
\mathbb{P}\left[\epsilon_{n}=1\right]=\mathbb{P}\left[\epsilon_{n}=-1\right]=\frac{1}{2} .
$$

It is well known that every martingale $\left(N_{n}\right)$ admits the unique "integral" representation:

$$
N_{n}=N_{0}+\sum_{k=1}^{n} h_{k}\left(\epsilon_{1}, \ldots, \epsilon_{k-1}\right) \epsilon_{k}
$$

for some functions $h_{k}=h_{k}\left(x_{1}, \ldots, x_{k-1}\right), k \geq 1$, where $h_{1}$ is just a constant.

Let $I=\left(x_{n}\right)$ be an arbitrary sequence in $\mathbf{R}$. We define a linear map $x \mapsto \xi(x)$ from $\mathbf{R}$ to $\mathcal{L}_{\infty}(\mathbf{R})$ by

$$
\xi(x)=\sum_{n=1}^{\infty} \frac{\left(x-x_{n}\right)}{2^{n}\left(1+\left|x_{n}\right|\right)} \epsilon_{n}=\psi_{0}+\psi_{1} x,
$$

where $\psi_{0}$ and $\psi_{1}$ are bounded random variables:

$$
\psi_{0}=-\sum_{n=1}^{\infty} \frac{x_{n}}{2^{n}\left(1+\left|x_{n}\right|\right)} \epsilon_{n}, \quad \psi_{1}=\sum_{n=1}^{\infty} \frac{1}{2^{n}\left(1+\left|x_{n}\right|\right)} \epsilon_{n} .
$$


We have that

$$
M_{n}(x)=\mathbb{E}\left[\xi(x) \mid \mathcal{F}_{n}\right]=\mathbb{E}\left[\xi(x) \mid \epsilon_{1}, \ldots, \epsilon_{n}\right]=\sum_{k=1}^{n} \frac{\left(x-x_{k}\right)}{2^{k}\left(1+\left|x_{k}\right|\right)} \epsilon_{k}
$$

and thus

$$
\Delta M_{n}(x)=M_{n}(x)-M_{n-1}(x)=\frac{\left(x-x_{n}\right)}{2^{n}\left(1+\left|x_{n}\right|\right)} \epsilon_{n} .
$$

If $x \notin I$, then the martingale $\left(N_{n}\right)$ from (3) is a stochastic integral with respect to $M(x)$ :

$$
N_{n}=N_{0}+\sum_{k=1}^{n} h_{k}\left(\epsilon_{1}, \ldots, \epsilon_{k-1}\right) \frac{2^{k}\left(1+\left|x_{k}\right|\right)}{\left(x-x_{k}\right)} \Delta M_{k}(x) .
$$

However, if $x_{m} \in I$, then the martingales $M\left(x_{m}\right)$ and

$$
L_{n}^{(m)}=\sum_{k=1}^{n} 1_{\{k=m\}} \epsilon_{k}=1_{\{n \geq m\}} \epsilon_{m}, \quad n \geq 0,
$$

are orthogonal. Hence, $L^{(m)}$ does not admit an integral representation with respect to $M\left(x_{m}\right)$.

The rest of the section is devoted to the proof of Theorem 3.1. It relies on Theorems A.1 and B.1 from the appendices and on the lemmas below.

Throughout the paper all operations on stochastic processes are defined pointwise, for every $(t, \omega)$. In particular, if $X$ is a matrix-valued process, then $|X|$ denotes the one-dimensional process of the running norm:

$$
|X|_{t}(\omega) \triangleq\left|X_{t}(\omega)\right|
$$

Let $X$ be a (uniformly) square integrable martingale taking values in $\mathbf{R}^{m}$. We denote by $[X]=\left(\left[X^{i}, X^{j}\right]\right)$ its process of quadratic variation and by $\langle X\rangle=\left(\left\langle X^{i}, X^{j}\right\rangle\right)$ its predictable process of quadratic variation; they both take values in the cone $\mathcal{S}_{+}^{m}$ of symmetric nonnegative $m \times m$-matrices. We define the predictable increasing process

$$
A^{X} \triangleq \operatorname{tr}\langle X\rangle=\sum_{i=1}^{m}\left\langle X^{i}, X^{i}\right\rangle
$$


Standard arguments show that there is a predictable process $\kappa^{X}$ with values in $\mathcal{S}_{+}^{m}$ such that

$$
\langle X\rangle=\left(\kappa^{X}\right)^{2} \cdot A^{X} .
$$

On the predictable $\sigma$-algebra $\mathcal{P}$ of $[0, \infty) \times \Omega$ we introduce a measure

$$
\mu^{X}(d t, d \omega) \triangleq d A_{t}^{X}(\omega) \mathbb{P}[d \omega]
$$

For a nonnegative predictable process $\gamma$ the expectation under $\mu^{X}$ is given by

$$
\mathbb{E}^{\mu^{X}}[\gamma]=\mathbb{E}\left[\int_{0}^{\infty} \gamma d A^{X}\right]=\mathbb{E}\left[\int_{0}^{\infty} \gamma_{t} d A_{t}^{X}\right]
$$

We observe that this measure is finite:

$$
\mu^{X}([0, \infty) \times \Omega)=\mathbb{E}\left[A_{\infty}^{X}\right]=\mathbb{E}\left[\left|X_{\infty}-X_{0}\right|^{2}\right]<\infty
$$

For predictable $m$-dimensional processes $\left(\gamma^{n}\right)$ and $\gamma$ the notation $\gamma^{n} \stackrel{\mu^{X}}{\rightarrow} \gamma$ stands for the convergence in measure $\mu^{X}$ :

$$
\forall \epsilon>0: \quad \mu^{X}\left[\left|\gamma^{n}-\gamma\right|>\epsilon\right] \rightarrow 0, \quad n \rightarrow \infty
$$

Lemma 3.3. Let $X$ be a square integrable martingale with values in $\mathbf{R}^{m}$ and $\gamma$ be a predictable $m$-dimensional process. Then $\gamma$ is $X$-integrable and $\gamma \cdot X=0$ if and only if $\kappa^{X} \gamma=0, \mu^{X}-$ a.s..

Proof. Since $\gamma 1_{\{|\gamma| \leq n\}} \cdot X \rightarrow \gamma \cdot X$ as $n \rightarrow \infty$ in the semimartingale topology, we can assume without a loss in generality that $\gamma$ is bounded. Then $\gamma \cdot X$ is a square integrable martingale with predictable quadratic variation

$$
\langle\gamma \cdot X\rangle_{t}=\int_{0}^{t}\left|\kappa^{X} \gamma\right|^{2} d A^{X}=\int_{0}^{t}\left|\kappa_{s}^{X} \gamma_{s}\right|^{2} d A_{s}^{X}
$$

and the result follows from the identity:

$$
\mathbb{E}\left[(\gamma \cdot X)_{\infty}^{2}\right]=\mathbb{E}\left[\langle\gamma \cdot X\rangle_{\infty}\right]=\mathbb{E}\left[\int_{0}^{\infty}\left|\kappa^{X} \gamma\right|^{2} d A^{X}\right]=\mathbb{E}^{\mu^{X}}\left[\left|\kappa^{X} \gamma\right|^{2}\right]
$$


For every predictable process $\zeta$ taking values in $\mathcal{S}_{+}^{m}$ we can naturally define a $\mathcal{S}_{+}^{m}$-valued predictable process $\zeta^{\oplus}$ such that for all $(t, \omega)$ the matrix $\zeta_{t}^{\oplus}(\omega)$ is the pseudo-inverse to the matrix $\zeta_{t}(\omega)$.

From Lemma 3.3 we deduce that if $\alpha$ is an integrand for $X$ then the predictable process

$$
\beta \triangleq \kappa^{X^{\oplus}} \kappa^{X} \alpha
$$

is also $X$-integrable and $\alpha \cdot X=\beta \cdot X$. Moreover, $|\beta| \leq|\alpha|$, by the minimal norm property of the pseudo-inverse matrices. In view of this property, we call a predictable $m$-dimensional process $\gamma$ a minimal integrand for $X$ if $\gamma$ is $X$-integrable and

$$
\gamma=\kappa^{X \oplus} \kappa^{X} \gamma
$$

From the definition of a minimal integrand we immediately deduce that

$$
\left|\kappa^{X} \gamma\right| \leq\left|\kappa^{X}\right||\gamma|, \quad|\gamma| \leq\left|\kappa^{X^{\oplus}}\right|\left|\kappa^{X} \gamma\right|
$$

where, following our convention, both the norm and the inequalities are defined pointwise, for every $(t, \omega)$.

We denote by $\mathcal{H}_{1}=\mathcal{H}_{1}\left(\mathbf{R}^{d}\right)$ the Banach space of uniformly integrable $d$-dimensional martingales $M$ with the norm:

$$
\|M\|_{\mathcal{H}_{1}} \triangleq \mathbb{E}\left[\sup _{t \geq 0}\left|M_{t}\right|\right] .
$$

By Davis' inequality, the convergence $M^{n} \rightarrow 0$ in $\mathcal{H}_{1}$ is equivalent to the convergence $\left[M^{n}\right]_{\infty}^{1 / 2} \rightarrow 0$ in $\mathcal{L}_{1}$, where $\left[M^{n}\right]$ is the quadratic variation process of $M^{n}$.

We say that a sequence $\left(N^{n}\right)$ of local martingales converges to a local martingale $N$ in $\mathcal{H}_{1, l o c}$ if there are stopping times $\left(\tau^{m}\right)$ such that $\tau^{m} \uparrow \infty$ and $N^{n, \tau^{m}} \rightarrow N^{\tau^{m}}$ in $\mathcal{H}_{1}$. Here as usual, we write $Y^{\tau} \triangleq\left(Y_{\min (t, \tau)}\right)$ for a semimartingale $Y$ stopped at a stopping time $\tau$.

Lemma 3.4. Let $X$ be a square integrable martingale with values in $\mathbf{R}^{m}$ and $\left(\gamma^{n}\right)$ be a sequence of predictable $m$-dimensional $X$-integrable processes such that the stochastic integrals $\left(\gamma^{n} \cdot X\right)$ converge to 0 in $\mathcal{H}_{1, \text { loc }}$. Then $\kappa^{X} \gamma^{n} \stackrel{\mu^{X}}{\rightarrow} 0$. If, in addition, $\left(\gamma^{n}\right)$ are minimal integrands then $\gamma^{n} \stackrel{\mu^{X}}{\rightarrow} 0$.

Proof. It is sufficient to consider the case of minimal integrands. By localization, we can suppose that $\gamma^{n} \cdot X \rightarrow 0$ in $\mathcal{H}_{1}$, which by Davis' inequality is equivalent to the convergence of $\left(\left[\gamma^{n} \cdot X\right]_{\infty}^{1 / 2}\right)$ to 0 in $\mathcal{L}_{1}$. 
Assume for a moment that $\left|\gamma^{n}\right| \leq 1$. Then $\left[\gamma^{n} \cdot X\right] \leq[X]$ and the theorem of dominated convergence yields that $\left[\gamma^{n} \cdot X\right]_{\infty} \rightarrow 0$ in $\mathcal{L}_{1}$. As

$$
\mathbb{E}\left[\left[\gamma^{n} \cdot X\right]_{\infty}\right]=\mathbb{E}\left[\left\langle\gamma^{n} \cdot X\right\rangle_{\infty}\right]=\mathbb{E}\left[\int_{0}^{\infty}\left|\kappa^{X} \gamma^{n}\right|^{2} d A^{X}\right]=\mathbb{E}^{\mu^{X}}\left[\left|\kappa^{X} \gamma^{n}\right|^{2}\right],
$$

we deduce that $\kappa^{X} \gamma^{n} \stackrel{\mu^{X}}{\rightarrow} 0$, which in view of (4), also implies that $\gamma^{n} \stackrel{\mu^{X}}{\rightarrow} 0$.

In the general case, we observe that

$$
\beta^{n} \triangleq \frac{1}{1+\left|\gamma^{n}\right|} \gamma^{n}
$$

are minimal integrands for $X$ such that $\left|\beta^{n}\right| \leq 1$ and $\left[\beta^{n} \cdot X\right] \leq\left[\gamma^{n} \cdot X\right]$. Hence, by what we have already proved, $\beta^{n} \stackrel{\mu^{X}}{\rightarrow} 0$, which clearly yields that $\gamma^{n} \stackrel{\mu^{X}}{\rightarrow} 0$ and then that $\kappa^{X} \gamma^{n} \stackrel{\mu^{X}}{\rightarrow} 0$.

Lemma 3.5. Let $X$ be a square integrable $m$-dimensional martingale and $\gamma=\left(\gamma^{i j}\right)$ be a predictable $X$-integrable process with values in $\mathbf{R}^{m \times d}$. Then $X$ is a stochastic integral with respect to $Y \triangleq \gamma \cdot X$, that is $X=X_{0}+\zeta \cdot Y$ for some predictable $Y$-integrable $d \times m$-dimensional process $\zeta$, if and only if

$$
\operatorname{rank} \kappa^{X} \gamma=\operatorname{rank} \kappa^{X}, \quad \mu^{X}-\text { a.s.. }
$$

Proof. We recall that a predictable process $\zeta$ is $Y=\gamma \cdot X$-integrable if and only if $\gamma \zeta$ is $X$-integrable. From Lemma 3.3 we deduce that $\zeta$ is $Y$-integrable and satisfies

$$
X=X_{0}+\zeta \cdot Y=X_{0}+\zeta \cdot(\gamma \cdot X)=(\gamma \zeta) \cdot X
$$

if and only if

$$
\kappa^{X} \gamma \zeta=\kappa^{X}, \quad \mu^{X}-\text { a.s.. }
$$

However, the solvability of this linear equation with respect to $\zeta$ is equivalent to (5) by an elementary argument from linear algebra.

Lemma 3.6. Let $U$ be an open connected set in $\mathbf{R}^{d}$ and $x \mapsto \sigma(x)$ be an analytic map with values in $k \times l$-matrices. Then there is a nonzero realanalytic function $f$ on $U$ such that

$$
E \triangleq\left\{x \in U: \operatorname{rank} \sigma(x)<\sup _{y \in U} \operatorname{rank} \sigma(y)\right\}=\{x \in U: f(x)=0\} .
$$

In particular, the set $E$ has Lebesgue measure zero and if $d=1$, then it consists of isolated points. 
Proof. Let $m \triangleq \sup _{y \in U} \operatorname{rank} \sigma(y)$. If $m=0$, then the set $E$ is empty and we can take $f=1$. If $m>0$, then the result holds for

$$
f(x)=\sum_{\alpha} \operatorname{det} \sigma_{\alpha}(x) \sigma_{\alpha}^{*}(x),
$$

where $\left(\sigma_{\alpha}\right)$ is the family of all $m \times m$ sub-matrices of $\sigma$. The remaining assertions follow from the well-known properties of zero-sets of real-analytic functions.

Proof of Theorem 3.1. Without restricting generality we can assume that $\zeta\left(x_{0}\right)=1$ and, hence, $\mathbb{Q}\left(x_{0}\right)=\mathbb{P}$. Proposition 2 in [9] shows that if some multi-dimensional local martingale has the MRP, then there is a bounded, hence square integrable, $m$-dimensional martingale $X$ that has the MRP. We fix such $X$ and use for it the $\mathcal{S}_{+}^{m}$-valued predictable process $\kappa^{X}$ and the finite measure $\mu^{X}$ on the predictable $\sigma$-algebra $\mathcal{P}$ introduced just before Lemma 3.3.

We define the martingales

$$
Y_{t}(x) \triangleq \mathbb{E}\left[\zeta(x) \mid \mathcal{F}_{t}\right], \quad R_{t}(x) \triangleq \mathbb{E}\left[\xi(x) \mid \mathcal{F}_{t}\right],
$$

and observe that $R(x)=S(x) Y(x)$. Let $\alpha(x)$ and $\beta(x)$ be integrands for $X$ with values in $\mathbf{R}^{m}$ and $\mathbf{R}^{m \times d}$, respectively, such that

$$
\begin{aligned}
& Y(x)=Y_{0}(x)+Y_{-}(x) \alpha(x) \cdot X, \\
& R(x)=R_{0}(x)+Y_{-}(x) \beta(x) \cdot X,
\end{aligned}
$$

where as usual, $Y_{-}$stands for the left-continuous process $\left(Y_{t-}\right)$. Integration by parts yields that

$$
d R(x)-S_{-}(x) d Y(x)=Y_{-}(x) d(S(x)+[S(x), \alpha(x) \cdot X]) .
$$

It follows that

$$
S(x)+[S(x), \alpha(x) \cdot X]=S_{0}(x)+\sigma(x) \cdot X,
$$

where

$$
\sigma(x)=\beta(x)-\alpha(x) S_{-}^{*}(x) .
$$


From Theorem B.1 we deduce that $S(x)$ has the MRP (under $\mathbb{Q}(x)$ ) if and only if the stochastic integral $\sigma(x) \cdot X$ has the MRP. By Lemma 3.5 the latter property is equivalent to

$$
\operatorname{rank} \kappa^{X} \sigma(x)=\operatorname{rank} \kappa^{X}, \quad \mu^{X}-\text { a.s. }
$$

and therefore, the exception set $I$ admits the description:

$$
I=\left\{x \in U: \mu^{X}[D(x)]>0\right\},
$$

where for $x \in U \cup\left\{x_{0}\right\}$ the predictable set $D(x)$ is given by

$$
D(x)=\left\{(t, \omega): \operatorname{rank} \kappa_{t}^{X}(\omega) \sigma_{t}(x)(\omega)<\operatorname{rank} \kappa_{t}^{X}(\omega)\right\} .
$$

From Theorem A.1 we deduce the existence of the integrands $\alpha(x)$ and $\beta(x)$ and of the modifications of the martingales $Y(x)$ and $R(x)$ such that for every $(t, \omega) \in[0, \infty) \times \Omega$ the function

$$
x \mapsto \sigma_{t}(x)(\omega)=\beta_{t}(x)(\omega)-\alpha_{t}(x)(\omega) \frac{R_{t-}^{*}(x)(\omega)}{Y_{t-}(x)(\omega)},
$$

taking values in the space of $m \times d$-matrices, is analytic on $U$. Hereafter, we shall use these versions.

Let $\lambda$ be the Lebesgue measure on $\mathbf{R}^{l}$ and $\mathcal{B}=\mathcal{B}(U)$ be the Borel $\sigma$ algebra on $U$. Since for every $(t, \omega)$ the function $x \mapsto \sigma_{t}(x)(\omega)$ is continuous on $U$, the function $(t, \omega, x) \mapsto \sigma_{t}(x)(\omega)$ is $\mathcal{P} \times \mathcal{B}$-measurable. It follows that

$$
E \triangleq\left\{(t, \omega, x): \operatorname{rank} \kappa_{t}^{X}(\omega) \sigma_{t}(x)(\omega)<\operatorname{rank} \kappa_{t}^{X}(\omega)\right\} \in \mathcal{P} \times \mathcal{B} .
$$

From Fubini's theorem we deduce the equivalences:

$$
\left(\mu^{X} \times \lambda\right)[E]=0 \quad \Leftrightarrow \quad \mu^{X}[F]=0 \quad \Leftrightarrow \quad \lambda[I]=0
$$

where

$$
F \triangleq\left\{(t, \omega): \lambda\left[\left\{x \in U: \operatorname{rank} \kappa_{t}^{X}(\omega) \sigma_{t}(x)(\omega)<\operatorname{rank} \kappa_{t}^{X}(\omega)\right\}\right]>0\right\} .
$$

Hence to obtain the multi-dimensional version of the theorem we need to show that $\mu^{X}(F)=0$.

From Lemma 3.6 and the analyticity of the function $x \mapsto \sigma_{t}(x)(\omega)$ we deduce that

$$
F=\left\{(t, \omega): \operatorname{rank} \kappa_{t}^{X}(\omega) \sigma_{t}(x)(\omega)<\operatorname{rank} \kappa_{t}^{X}(\omega), \forall x \in U\right\} .
$$


We recall now that if $\left(x_{n}\right)$ is a sequence in $U$ that converges to $x_{0}$, then the martingales $\left(R\left(x_{n}\right), Y\left(x_{n}\right)\right)$ converge to the martingale $\left(R\left(x_{0}\right), Y\left(x_{0}\right)\right)=$ $\left(S\left(x_{0}\right), 1\right)$ in $\mathcal{L}_{1}$. By Lemma A.3, passing to a subsequence, we can assume that $\left(R\left(x_{n}\right), Y\left(x_{n}\right)\right) \rightarrow\left(R\left(x_{0}\right), Y\left(x_{0}\right)\right)$ in $\mathcal{H}_{1, \text { loc }}$. From Lemma 3.4 we deduce that

$$
\begin{aligned}
& \kappa^{X} \alpha\left(x_{n}\right) \stackrel{\mu^{X}}{\rightarrow} 0, \\
& \kappa^{X} \beta\left(x_{n}\right) \stackrel{\mu^{X}}{\rightarrow} \kappa^{X} \beta\left(x_{0}\right)=\kappa^{X} \sigma\left(x_{0}\right) .
\end{aligned}
$$

It follows that

$$
\kappa^{X} \sigma\left(x_{n}\right)=\kappa^{X}\left(\beta\left(x_{n}\right)-\alpha\left(x_{n}\right) S_{-}^{*}\left(x_{n}\right)\right) \stackrel{\mu^{X}}{\rightarrow} \kappa^{X} \beta\left(x_{0}\right)=\kappa^{X} \sigma\left(x_{0}\right) .
$$

Passing to a subsequence we can choose the sequence $\left(x_{n}\right)$ so that

$$
\kappa^{X} \sigma\left(x_{n}\right) \rightarrow \kappa^{X} \sigma\left(x_{0}\right), \quad \mu^{X}-a . s . .
$$

As $a \mapsto \operatorname{rank} a$ is a lower-semicontinuous function on matrices, it follows that

$$
\liminf _{n} \operatorname{rank} \kappa^{X} \sigma\left(x_{n}\right) \geq \operatorname{rank} \kappa^{X} \sigma\left(x_{0}\right), \quad \mu^{X}-\text { a.s.. }
$$

Accounting for (6) we obtain that

$$
F \subset D\left(x_{0}\right), \quad \mu^{X}-a . s . .
$$

However, as $S\left(x_{0}\right)$ has the MRP, Lemma 3.5 yields that $\mu^{X}\left[D\left(x_{0}\right)\right]=0$ and the multi-dimensional version of the theorem follows.

Assume now that $U$ is an open interval in $\mathbf{R}$ and that contrary to the assertion of the theorem the exception set $I$ is uncountable. Then there are $\epsilon>0$, a closed interval $[a, b] \subset U$, and a sequence $\left(x_{n}\right) \subset[a, b]$ such that

$$
\mu^{X}\left[D\left(x_{n}\right)\right] \geq \epsilon, \quad n \geq 1 .
$$

Since for every $(t, \omega)$ the function $x \mapsto \sigma_{t}(x)(\omega)$ is analytic, we deduce from Lemma 3.6 that on every closed interval the integer-valued function $x \mapsto$ $\operatorname{rank}\left(\kappa_{t}^{X}(\omega) \sigma_{t}(x)(\omega)\right)$ has constant value except for a finite number of points, where its values are smaller. Hence, if

$$
\operatorname{rank}\left(\kappa_{t}^{X}(\omega) \sigma_{t}\left(x_{n^{\prime}}\right)(\omega)\right)<\operatorname{rank}\left(\kappa_{t}^{X}(\omega)\right) \text { for countable }\left(n^{\prime}\right) \subset(n),
$$


then

$$
\operatorname{rank}\left(\kappa_{t}^{X}(\omega) \sigma_{t}(x)(\omega)\right)<\operatorname{rank}\left(\kappa_{t}^{X}(\omega)\right) \text { for all } x \in U
$$

Accounting for (6) it follows that

$$
\limsup _{n} D\left(x_{n}\right) \triangleq \cap_{n} \cup_{m \geq n} D\left(x_{m}\right)=F
$$

and thus

$$
\mu^{X}[F] \geq \limsup _{n} \mu^{X}\left[D\left(x_{n}\right)\right] \geq \epsilon .
$$

However, as we have already shown, $\mu^{X}[F]=0$ and we arrive to a contradiction.

\section{A Analytic fields of martingales and stochas- tic integrals}

We denote by $\mathbf{D}^{\infty}\left([0, \infty), \mathbf{R}^{d}\right)$ the Banach space of RCLL (right-continuous with left limits) functions $f:[0, \infty) \rightarrow \mathbf{R}^{d}$ equipped with the uniform norm: $\|f\|_{\infty} \triangleq \sup _{t \geq 0}|f(t)|$.

Theorem A.1. Let $U$ be an open connected set in $\mathbf{R}^{l}$ and $x \mapsto \xi(x)$ be an analytic map from $U$ to $\mathcal{L}_{1}\left(\mathbf{R}^{d}\right)$. Then there are modifications of the accompanying d-dimensional martingales

$$
M_{t}(x) \triangleq \mathbb{E}\left[\xi(x) \mid \mathcal{F}_{t}\right]
$$

such that for every $\omega \in \Omega$ the maps $x \mapsto M .(x)(\omega)$ taking values in $\mathbf{D}^{\infty}\left([0, \infty), \mathbf{R}^{d}\right)$ are analytic on $U$.

If in addition, the MRP holds for a local martingale $X$ with values in $\mathbf{R}^{m}$, then there is a stochastic field $x \mapsto \sigma(x)$ of integrands for $X$ such that

$$
M(x)=M_{0}(x)+\sigma(x) \cdot X,
$$

and for every $(t, \omega) \in[0, \infty) \times \Omega$ the function $x \mapsto \sigma_{t}(x)(\omega)$ taking values in $m \times d$-matrices is analytic on $U$.

The proof of the theorem is divided into a series of lemmas. For a multiindex $\alpha=\left(\alpha_{1}, \ldots, \alpha_{l}\right) \in \mathbf{Z}_{+}^{l}$ we denote

$$
|\alpha| \triangleq \alpha_{1}+\cdots+\alpha_{l} .
$$

The space $\mathcal{H}_{1}$ has been introduced just before Lemma 3.4. 
Lemma A.2. Let $\left(M^{\alpha}\right)_{\alpha \in \mathbf{Z}_{+}^{l}}$ be uniformly integrable martingales with values in $\mathbf{R}^{d}$ such that

$$
\sum_{\alpha} 2^{|\alpha|}\left\|M^{\alpha}\right\|_{\mathcal{L}_{1}}<\infty
$$

Then there is an increasing sequence $\left(\tau_{m}\right)$ of stopping times such that $\left\{\tau_{m}=\infty\right\} \uparrow$ $\Omega$ and

$$
\sum_{\alpha}\left\|M^{\alpha, \tau_{m}}\right\|_{\mathcal{H}_{1}}<\infty, \quad m \geq 1 .
$$

Proof. We define the martingale

$$
L_{t} \triangleq \mathbb{E}\left[\sum_{\alpha} 2^{|\alpha|}\left|M_{\infty}^{\alpha}\right| \mid \mathcal{F}_{t}\right], t \geq 0,
$$

and stopping times

$$
\tau_{m} \triangleq \inf \left\{t \geq 0: L_{t} \geq m\right\}, m \geq 1 .
$$

Clearly, $\left\{\tau_{m}=\infty\right\} \uparrow \Omega$ as $m \rightarrow \infty$ and $\left|M^{\alpha}\right| \leq 2^{-|\alpha|} L$. Moreover,

$$
\left\|L^{\tau_{m}}\right\|_{\mathcal{H}_{1}}=\mathbb{E}\left[\sup _{0 \leq t \leq \tau_{m}} L_{t}\right] \leq m+\mathbb{E}\left[L_{\tau_{m}}\right]=m+L_{0}<\infty .
$$

It follows that

$$
\sum_{\alpha}\left\|M^{\alpha, \tau_{m}}\right\|_{\mathcal{H}_{1}} \leq\left\|L^{\tau_{m}}\right\|_{\mathcal{H}_{1}} \sum_{\alpha} 2^{-|\alpha|}<\infty
$$

Lemma A.3. Let $\left(M^{n}\right)$ and $M$ be uniformly integrable martingales such that $M^{n} \rightarrow M$ in $\mathcal{L}_{1}$. Then there exists a subsequence of $\left(M^{n}\right)$ that converges to $M$ in $\mathcal{H}_{1, l o c}$.

Proof. Since $M^{n} \rightarrow M$ in $\mathcal{L}_{1}$ there exists a subsequence $\left(M^{n_{k}}\right)$ such that

$$
\sum_{k=1}^{\infty}\left\|M^{n_{k+1}}-M^{n_{k}}\right\|_{\mathcal{L}_{1}} 2^{k}<\infty
$$

Lemma A.2 implies that $M^{n_{k}} \rightarrow M$ in $\mathcal{H}_{1, l o c}$. 
Let $X$ be a square integrable martingale taking values in $\mathbf{R}^{m}$. As in Section 3 we associate with $X$ the increasing predictable process $A^{X} \triangleq \operatorname{tr}\langle X\rangle$, the $\mathcal{S}_{+}^{m}$-valued predictable process $\kappa^{X}$ such that $\langle X\rangle=\left(\kappa^{X}\right)^{2} \cdot A^{X}$, and a finite measure $\mu^{X}(d t, d \omega) \triangleq d A_{t}^{X}(\omega) \mathbb{P}[d \omega]$ on the predictable $\sigma$-algebra $\mathcal{P}$ of $[0, \infty) \times \Omega$. We recall that an integrand $\gamma$ for $X$ is minimal if

$$
\gamma=\kappa^{X^{\oplus}} \kappa^{X} \gamma
$$

Lemma A.4. Let $X$ be a bounded martingale with values in $\mathbf{R}^{m}$ and $\left(\gamma^{\alpha}\right)_{\alpha \in \mathbf{Z}_{+}^{l}}$ be minimal integrands for $X$ such that

$$
\sum_{\alpha}\left\|\gamma^{\alpha} \cdot X\right\|_{\mathcal{H}^{1}}<\infty
$$

Then

$$
\sum_{\alpha}\left|\gamma^{\alpha}\right|^{2}=\sum_{\alpha}\left|\gamma_{t}^{\alpha}(\omega)\right|^{2}<\infty, \quad \mu^{X}-\text { a.s.. }
$$

Proof. By Davis' inequality, (8) is equivalent to

$$
\sum_{\alpha} \mathbb{E}\left[\left[\gamma^{\alpha} \cdot X\right]_{\infty}^{1 / 2}\right]<\infty
$$

By replacing if necessary $\gamma^{\alpha}$ with $\frac{1}{1+\left|\gamma^{\alpha}\right|} \gamma^{\alpha}$, we can assume without a loss of generality that $\left|\gamma^{\alpha}\right| \leq 1$. Let us show that in this case the increasing optional process

$$
B_{t} \triangleq \sum_{\alpha}\left[\gamma^{\alpha} \cdot X\right]_{t}, \quad t \geq 0,
$$

is locally integrable. Since

$$
B_{\infty}=\sum_{\alpha}\left[\gamma^{\alpha} \cdot X\right]_{\infty} \leq\left(\sum_{\alpha}\left[\gamma^{\alpha} \cdot X\right]_{\infty}^{1 / 2}\right)^{2}<\infty,
$$

we only need to check that the positive jump process $\Delta B$ is locally integrable. Actually, we shall show that $\sup _{t \geq 0} \Delta B_{t}$ is integrable. Indeed, as $X$ is bounded, there is a constant $c>0$ such that $\left|\left(\gamma^{\alpha}\right)^{*} \Delta X\right| \leq c$. Hence,

$$
\sup _{t \geq 0} \Delta B_{t} \leq \sum_{\alpha}\left(\left(\gamma^{\alpha}\right)^{*} \Delta X\right)^{2} \leq c \sum_{\alpha}\left|\left(\gamma^{\alpha}\right)^{*} \Delta X\right| \leq c \sum_{\alpha}\left[\gamma^{\alpha} \cdot X\right]_{\infty}^{1 / 2},
$$

where the right-hand side has finite expected value. 
Since for every stopping time $\tau$

$$
\mathbb{E}\left[B_{\tau}\right]=\sum_{\alpha} \mathbb{E}\left[\left[\gamma^{\alpha} \cdot X\right]_{\tau}\right]=\sum_{\alpha} \mathbb{E}\left[\int_{0}^{\tau}\left|\kappa^{X} \gamma^{\alpha}\right|^{2} d A^{X}\right],
$$

the local integrability of $B$ yields the existence of stopping times $\left(\tau^{m}\right)$ such that $\tau_{m} \uparrow \infty$ and

$$
\sum_{\alpha} \mathbb{E}\left[\int_{0}^{\tau_{m}}\left|\kappa^{X} \gamma^{\alpha}\right|^{2} d A^{X}\right]=\sum_{\alpha} \mathbb{E}^{\mu^{X}}\left[\left|\kappa^{X} \gamma^{\alpha}\right|^{2} 1_{\left[0, \tau^{m}\right]}\right]<\infty .
$$

It follows that

$$
\sum_{\alpha}\left|\kappa^{X} \gamma^{\alpha}\right|^{2}<\infty, \quad \mu^{X}-a . s . .
$$

This convergence implies (9) in view of inequalities (4) for minimal integrands.

Lemma A.5. Let $X$ be a square integrable martingale taking values in $\mathbf{R}^{m}$ and $\left(\gamma^{n}\right)$ be minimal integrands for $X$ such that $\left(M^{n} \triangleq \gamma^{n} \cdot X\right)$ are uniformly integrable martingales. Suppose that there are a uniformly integrable martingale $M$ and a predictable process $\gamma$ such that $M^{n} \rightarrow M$ in $\mathcal{L}_{1}$ and $\gamma_{t}^{n}(\omega) \rightarrow \gamma_{t}(\omega)$ for every $(t, \omega)$. Then $\gamma$ is a minimal integrand for $X$ and $M=\gamma \cdot X$.

Proof. In view of characterization (7) for minimal integrands, the minimality of every element of $\left(\gamma^{n}\right)$ implies the minimality of $\gamma$ provided that the latter is $X$-integrable. Thus we only need to show that $\gamma$ is $X$-integrable and $M=\gamma \cdot X$.

By Lemma A.3, passing to subsequences, we can assume that $M^{n}=$ $\gamma^{n} \cdot X \rightarrow M$ in $\mathcal{H}_{1, l o c}$. Since the space of stochastic integrals is closed under the convergence in $\mathcal{H}_{1, l o c}$, there is a $X$-integrable predictable process $\widetilde{\gamma}$ such that $M=\widetilde{\gamma} \cdot X$. From Lemma 3.4 we deduce that

$$
\kappa^{X}\left(\gamma^{n}-\widetilde{\gamma}\right) \stackrel{\mu^{X}}{\rightarrow} 0
$$

It follows that

$$
\kappa^{X}(\widetilde{\gamma}-\gamma)=0, \quad \mu^{X}-a . s .
$$

and Lemma 3.3 yields the result. 
Proof of Theorem A.1. It is sufficient to prove the existence of the required analytic versions only locally, in a neighborhood of every $y \in U$. Hereafter, we fix $y \in U$. There are $\epsilon=\epsilon(y) \in(0,1)$ and a family $\left(\zeta_{\alpha}=\zeta_{\alpha}(y)\right)_{\alpha \in \mathbf{Z}_{+}^{l}}$ in $\mathcal{L}_{1}$ such that

$$
\begin{gathered}
\xi(x)=\xi(y)+\sum_{\alpha} \zeta_{\alpha}(x-y)^{\alpha}, \quad \max _{i}\left|x_{i}-y_{i}\right|<2 \epsilon, \\
\sum_{\alpha} \mathbb{E}\left[\left|\zeta_{\alpha}\right|\right](2 \epsilon)^{|\alpha|}<\infty
\end{gathered}
$$

where the first series converges in $\mathcal{L}_{1}$.

By taking conditional expectations with respect to $\mathcal{F}_{t}$ we obtain that

$$
M_{t}(x)=M_{t}(y)+\sum_{\alpha} L_{t}^{\alpha}(x-y)^{\alpha}, \quad \max _{i}\left|x_{i}-y_{i}\right|<2 \epsilon,
$$

where $L_{t}^{\alpha} \triangleq \mathbb{E}\left[\zeta_{\alpha} \mid \mathcal{F}_{t}\right]$ and the series converges in $\mathcal{L}_{1}$. Lemma A.2 yields an increasing sequence $\left(\tau_{m}\right)$ of stopping times such that $\left\{\tau_{m}=\infty\right\} \uparrow \Omega$ and

$$
\sum_{\alpha}\left\|L^{\alpha, \tau_{m}}\right\|_{\mathcal{H}_{1}} \epsilon^{|\alpha|}<\infty, \quad m \geq 1
$$

It follows that

$$
\sum_{\alpha} \sup _{t \geq 0}\left|L_{t}^{\alpha}(\omega)\right| \epsilon^{|\alpha|}<\infty, \quad \mathbb{P}-\text { a.s. }
$$

and we can modify the martingales $\left(L^{\alpha}\right)$ so that the above convergence holds true for every $\omega \in \Omega$. Then the series in (10) converges uniformly in $t$ for every $\omega \in \Omega$ and every $x$ such that $\max _{i}\left|x_{i}-y_{i}\right|<\epsilon$. Thus, it defines the modifications of $M(x)$ for such $x$ with the required analytic properties.

For the second part of the theorem we observe that the statement is invariant with respect to the choice of the local martingale $X$ that has the MRP. Proposition 2 in [9] shows that we can choose $X$ to be a bounded $m$-dimensional martingale.

As $X$ has the MRP, there are minimal integrands $\sigma(y)$ and $\left(\gamma^{\alpha}\right)$ such that

$$
\begin{aligned}
M(y) & =M_{0}(y)+\sigma(y) \cdot X, \\
L^{\alpha} & =L_{0}^{\alpha}+\gamma^{\alpha} \cdot X, \quad \alpha \in \mathbf{Z}_{+}^{l} .
\end{aligned}
$$

From Lemma A.4 we deduce that

$$
\sum_{\alpha}\left|\gamma_{t}^{\alpha}(\omega)\right|^{2} \epsilon^{2|\alpha|}<\infty
$$


for all $(t, \omega)$ except a predictable set of $\mu^{X}$-measure 0. By Lemma 3.3 we can set $\gamma^{\alpha}=0$ on this set without changing $\gamma^{\alpha} \cdot X$. Then the series converges for every $(t, \omega)$. As $\epsilon \in(0,1)$, we deduce that

$$
\sum_{\alpha}\left|\gamma_{t}^{\alpha}(\omega)\right| \epsilon^{2|\alpha|}<\infty
$$

and thus for $x=\left(x_{1}, \ldots, x_{l}\right)$ such that $\max _{i}\left|x_{i}-y_{i}\right|<\epsilon^{2}$ and every $(t, \omega)$ we can define

$$
\sigma_{t}(x)(\omega) \triangleq \sigma_{t}(y)(\omega)+\sum_{\alpha} \gamma_{t}^{\alpha}(\omega)(x-y)^{\alpha}
$$

By construction, the function $x \rightarrow \sigma_{t}(x)(\omega)$ is analytic in a neighborhood of $y$. By Lemma A.5, for every $x$ such that $\max _{i}\left|x_{i}-y_{i}\right|<\epsilon^{2}$ the predictable process $\sigma(x)$ is an integrand for $X$ and

$$
\begin{aligned}
M(x) & =M(y)+\sum_{\alpha} L^{\alpha}(x-y)^{\alpha} \\
& =M_{0}(x)+\sigma(y) \cdot X+\sum_{\alpha}\left(\gamma^{\alpha} \cdot X\right)(x-y)^{\alpha} \\
& =M_{0}(x)+\sigma(x) \cdot X .
\end{aligned}
$$

\section{B The MRP under the change of measure}

Let $X$ be a $d$-dimensional local martingale and $Z>0$ be the density process of $\widetilde{\mathbb{P}} \sim \mathbb{P}$. We denote by $\widetilde{Z} \triangleq 1 / Z$ the density process of $\mathbb{P}$ under $\widetilde{\mathbb{P}}$ and set $L \triangleq \widetilde{Z}_{-} \cdot Z$ and $\widetilde{L} \triangleq Z_{-} \cdot \widetilde{Z}$. Using integration by parts we deduce that

$$
d(\widetilde{Z} X)=X_{-} d \widetilde{Z}+\widetilde{Z}_{-} d \widetilde{X}
$$

where

$$
\widetilde{X}=X+[X, \widetilde{L}]
$$

It follows that $\tilde{X}$ is a $d$-dimensional local martingale under $\widetilde{\mathbb{P}}$. Of course, this is just a version of Girsanov's theorem. 
We observe that the relations between $X$ and $\widetilde{X}$ are symmetric in the sense that

$$
X=\widetilde{X}+[\widetilde{X}, L] .
$$

Indeed, as we have already shown, $Y \triangleq \widetilde{X}+[\widetilde{X}, L]$ is a $d$-dimensional local martingale. Clearly, the local martingales $X$ and $Y$ have the same initial values and the same continuous martingale parts. Finally, they have identical jumps:

$$
\begin{aligned}
\Delta(Y-X) & =\Delta([X, \widetilde{L}]+[\widetilde{X}, L])=\Delta X(\Delta \widetilde{L}+\Delta L+\Delta \widetilde{L} \Delta L) \\
& =\Delta X \Delta(Z \widetilde{Z})=0 .
\end{aligned}
$$

Theorem B.1. The local martingale $X$ has the MRP if and only if the local martingale $\widetilde{X}$ under $\widetilde{\mathbb{P}}$ has the $M R P$.

Proof. By symmetry, it is sufficient to prove only one of the implications. We assume that $X$ has the MRP. Let $\widetilde{M}$ be a local martingale under $\widetilde{\mathbb{P}}$. The arguments before the statement of the theorem yield the unique local martingale $M$ such that

$$
\widetilde{M}=M+[M, \widetilde{L}] .
$$

If now $H$ is an integrand for $X$ such that $M=M_{0}+H \cdot X$, then

$$
\widetilde{M}=\widetilde{M}_{0}+H \cdot(X+[X, \widetilde{L}])=\widetilde{M}_{0}+H \cdot \widetilde{X} .
$$

\section{References}

[1] Robert M. Anderson and Roberto C. Raimondo. Equilibrium in continuous-time financial markets: endogenously dynamically complete markets. Econometrica, 76(4):841-907, 2008. ISSN 0012-9682.

[2] Mark Davis and Jan Obłój. Market completion using options. In Advances in mathematics of finance, volume 83 of Banach Center Publ., pages 49-60. Polish Acad. Sci. Inst. Math., Warsaw, 2008. doi: 10.4064/bc83-0-4. URL http://dx.doi.org/10.4064/bc83-0-4. 
[3] David German. Pricing in an equilibrium based model for a large investor. Math. Financ. Econ., 4(4):287-297, 2011. ISSN 1862-9679. doi: 10.1007/s11579-011-0041-6. URL http://dx.doi.org/10.1007/s11579-011-0041-6.

[4] J. Hugonnier, S. Malamud, and E. Trubowitz. Endogenous completeness of diffusion driven equilibrium markets. Econometrica, 80(3): 1249-1270, 2012. ISSN 1468-0262. doi: 10.3982/ECTA8783. URL http://dx.doi.org/10.3982/ECTA8783.

[5] Jean Jacod. Calcul stochastique et problèmes de martingales, volume 714 of Lecture Notes in Mathematics. Springer, Berlin, 1979. ISBN 3-540-09253-6.

[6] Dmitry Kramkov. Existence of an endogenously complete equilibrium driven by a diffusion. Finance Stoch., 19(1):1-22, 2015. ISSN 0949-2984. doi: 10.1007/s00780-014-0250-y. URL http://dx.doi.org/10.1007/s00780-014-0250-y.

[7] Dmitry Kramkov and Silviu Predoiu. Integral representation of martingales motivated by the problem of endogenous completeness in financial economics. Stochastic Processes and their Applications, 124(1): 81-100, 2014. ISSN 0304-4149. doi: 10.1016/j.spa.2013.06.017. URL http://dx.doi.org/10.1016/j.spa.2013.06.017.

[8] Dmitry Kramkov and Sergio Pulido. A system of quadratic BSDEs arising in a price impact model. Ann. Appl. Probab., 26(2):794-817, 2016. doi: 10.1214/15-AAP1103. URL http://dx.doi.org/10.1214/15-AAP1103.

[9] Dmitry Kramkov and Mihai Sîrbu. On the two-times differentiability of the value functions in the problem of optimal investment in incomplete markets. Ann. Appl. Probab., 16(3):1352-1384, 2006. ISSN 1050-5164.

[10] Frank Riedel and Frederik Herzberg. Existence of financial equilibria in continuous time with potentially complete markets. J. Math. Econom., 49(5):398-404, 2013. ISSN 0304-4068. doi: 10.1016/j.jmateco.2013.07. 001. URL http://dx.doi.org/10.1016/j.jmateco.2013.07.001.

[11] Daniel C. Schwarz. Market completion with derivative securities. Finance and Stochastics, 21(1):263-284, 2017. 
ISSN 1432-1122. doi: 10.1007/s00780-016-0317-z. URL https : //doi.org/10.1007/s00780-016-0317-z. 